\title{
Urinary netrin-1 concentration in healthy full-term newborns
}

\author{
Monika Kamianowska ${ }^{1}$, Marek Szczepański ${ }^{1}$, Elżbieta E. Kulikowska ${ }^{1}$, Barbara Bebko ${ }^{1}$, \\ Alicja Koput², Anna Wasilewska ${ }^{3}$
}

\author{
${ }^{1}$ Department of Neonatology and Neonatal Intensive Care, Medical University \\ of Bialystok, Bialystok, Poland \\ ${ }^{2}$ Department of Pediatric Laboratory Diagnostics, Medical University of Bialystok, \\ Bialystok, Poland \\ ${ }^{3}$ Department of Pediatrics and Nephrology, Medical University of Bialystok, Bialystok, \\ Poland
}

Submitted: 4 January 2018

Accepted: 28 March 2018

Arch Med Sci 2021; 17 (1): 47-52

DOI: https://doi.org/10.5114/aoms/89591

Copyright $\odot 2020$ Termedia \& Banach

\begin{abstract}
Introduction: Monitoring of renal function in acute kidney injury in the pediatric population is complicated by the lack of age-related reference values of new biomarkers. Urinary netrin-1 is a new marker to demonstrate early kidney damage. Netrin-1 has a molecular mass of $72 \mathrm{kDa}$. It is therefore unlikely that it is filtered by the glomerulus under normal conditions. However, netrin-1 is highly induced after acute and chronic kidney injury and excreted in urine in humans. The aim of the study was to determine the normal concentrations of urinary netrin-1 in healthy full-term newborns.

Material and methods: The study included 88 healthy full-term neonates (51 boys and 37 girls) born from normal, uncomplicated pregnancies. The concentration of netrin-1 was determined in urine obtained on the first or second day of life with a commercially available ELISA kit.

Results: The urinary concentration of netrin-1 in newborns was independent of gender and time of urine collection. We found a negative correlation between both the urinary netrin-1 concentration and urinary netrin-1 concentration after normalization for urinary creatinine and the birth weight.

Conclusions: This is the first study showing the urinary netrin-1 concentration in healthy full-term newborns. Future investigation is needed to confirm its potential role as a marker of kidney function in this age group.
\end{abstract}

Key words: healthy, netrin-1, newborn, normal ranges.

\section{Introduction}

Netrin-1 is a laminin-related secreted protein that was originally identified as axonal guidance cues [1]. It exerts its effects through two known receptors, deleted in colon cancer (DCC) and uncoordinated-5 (UNC5). Netrin-1 is widely expressed in many tissues, including renal tissues. It was initially described in the central nervous system (CNS) during neurogenesis [2]. In recent studies, it was documented that netrin-1 promoted angiogenesis, cell migration, and tissue morphogenesis, and played a significant role in regulation of inflammation $[3,4]$. In the kidneys, netrin-1 is expressed in endothelial cells in the normal kidney, and in tubular epithelial cells [5]. Netrin-1 has a molecular mass of $72 \mathrm{kDa}$. It is therefore

\author{
Corresponding author: \\ Monika Kamianowska MD \\ Department \\ of Neonatology and \\ Neonatal Intensive Care \\ Medical University \\ in Białystok \\ 24a M. C. Skłodowskiej St \\ 15-276 Białystok, Poland \\ Phone: +48 857468498 \\ Fax: +48 857468663 \\ E-mail: monikakamm@wp.pl
}


unlikely that it is filtered by the glomerulus under normal conditions [6]. However, netrin-1 is highly induced after acute and chronic kidney injury and excreted in urine in both mice and humans $[5,7]$.

Surprisingly, after injury, netrin-1 protein expression appears in proximal tubular epithelial cells but is downregulated in vascular endothelial cells [5]. What is more, ischemia/reperfusion (I/R) injury of the kidney leads to downregulation of netrin-1 mRNA in the peritubular capillaries and infiltration of leukocytes, normally inhibited by the presence of netrin-1, resulting in preventing renal dysfunction and epithelial cell apoptosis in response to ischemia $[5,7]$. In the light of these data, netrin-1 expression seems to be effective in preventing acute kidney injury (AKI). The fact that netrin-1 is a protein secreted in the urine has led to the discovery of netrin- 1 as an early diagnostic marker of kidney damage $[4,5]$. Elevated concentrations of urinary netrin- 1 have been well documented in normoalbuminuric diabetic adult patients when compared to healthy controls and still further elevated in patients with microalbuminuria and overt nephropathy [8]. It was also shown that increased urinary netrin-1 concentration precedes the onset of albuminuria in normotensive and normoalbuminuric obese children. Urinary netrin-1 excretion was shown to be influenced by insulin resistance and hyperinsulinemia [9].

Neonatal kidneys are more susceptible to injury due to their immaturity. This phenomenon is caused by a low glomerular filtration rate, high renal vascular resistance, high plasma renin activity, decreased intercortical perfusion and decreased reabsorption of sodium in the proximal tubules [10]. Clinical manifestations are even more evident in prematures, mainly because of the limited response of the kidney to aldosterone. The transient tubulopathy of prematurity, defined as "leaky tubules", results in inadequate handling of free water, electrolytes, small proteins and bicarbonate [11].

What is even more noteworthy is the fact that the urinary netrin-1 concentrations seem to be a marker of perinatal asphyxia [12]. Significantly, higher urinary concentrations of netrin-1 within $48 \mathrm{~h}$ after birth were found in neonates with acute renal injury than in the non-AKI group [13].

To the best of our knowledge, data on reference values of urinary netrin-1 concentrations in healthy full-term neonates have not been published so far.

\section{Material and methods}

\section{Patient recruitment}

This prospective study included 88 healthy fullterm neonates born at the Department of Perina- tology, and then hospitalized at the rooming-in ward at the Department of Neonatology and Intensive Neonatal Care, Medical University of Bialystok, Poland between September 2014 and August 2015. All newborns were the result of normal pregnancies without any prenatal and perinatal complications (Apgar score of more than $\geq 8$ in $1^{\text {st }}$ minute of birth). Subjects enrolled in this study met the following criteria: appropriate and constitutionally large weight for gestational age (GA), normal prenatal ultrasound examination of the kidney and normal physical examination. Newborns who had a history of any congenital anomaly, intrauterine growth retardation, heart disease, respiratory disorders, intrauterine infections, or elevated inflammatory markers were excluded from the analysis (Figure 1).

The study had approval from the Local Bioethics Committee at the Medical University of Bialystok. All the procedures were conducted in accordance with the Declaration of Helsinki. Prior to the study, written informed consent was obtained from parents of all the neonates.

\section{Sample collection}

In the study group, the urine samples were collected using single sterile bags (UNIDEM, London, UK) for newborns on the first or second day of life. Newborns were assigned randomly to groups in which urine was collected once on the first or second day of life. The blood samples were collected during routine practice in the unit. We used S-Monovette $1.2 \mathrm{ml}$, Clotting Activator/Serum test tubes (Sarstedt AG \& Co, North Rhine-Westphalia, Germany) for venous blood sampling. We took the sample of venous blood once, $1.2 \mathrm{ml}$ on the first or second day of life, depending on whether the newborn's urine was collected. The serum and urine samples obtained after centrifugation were kept in the fridge $\left(4^{\circ} \mathrm{C}\right)$ for a maximum of $2 \mathrm{~h}$, then frozen at $-80^{\circ} \mathrm{C}$, and stored for up to 4 months. We did not use repeated freeze-thaw cycles. The above analysis has taken into account the gender and time of sample collection.

\section{Determination of urinary netrin-1 and urinary creatinine}

Urinary concentrations of netrin-1 were determined with a commercially available ELISA kit from USCNLIFE (Wuhan EIAab Science Co., Ltd. China) according to the manufacturer's instructions. Briefly, a biotin-conjugated polyclonal antibody specific for netrin-1 was used to detect this marker in the analyzed material. Horseradish peroxidase-conjugated avidin was added, followed by a color-forming peroxidase substrate 


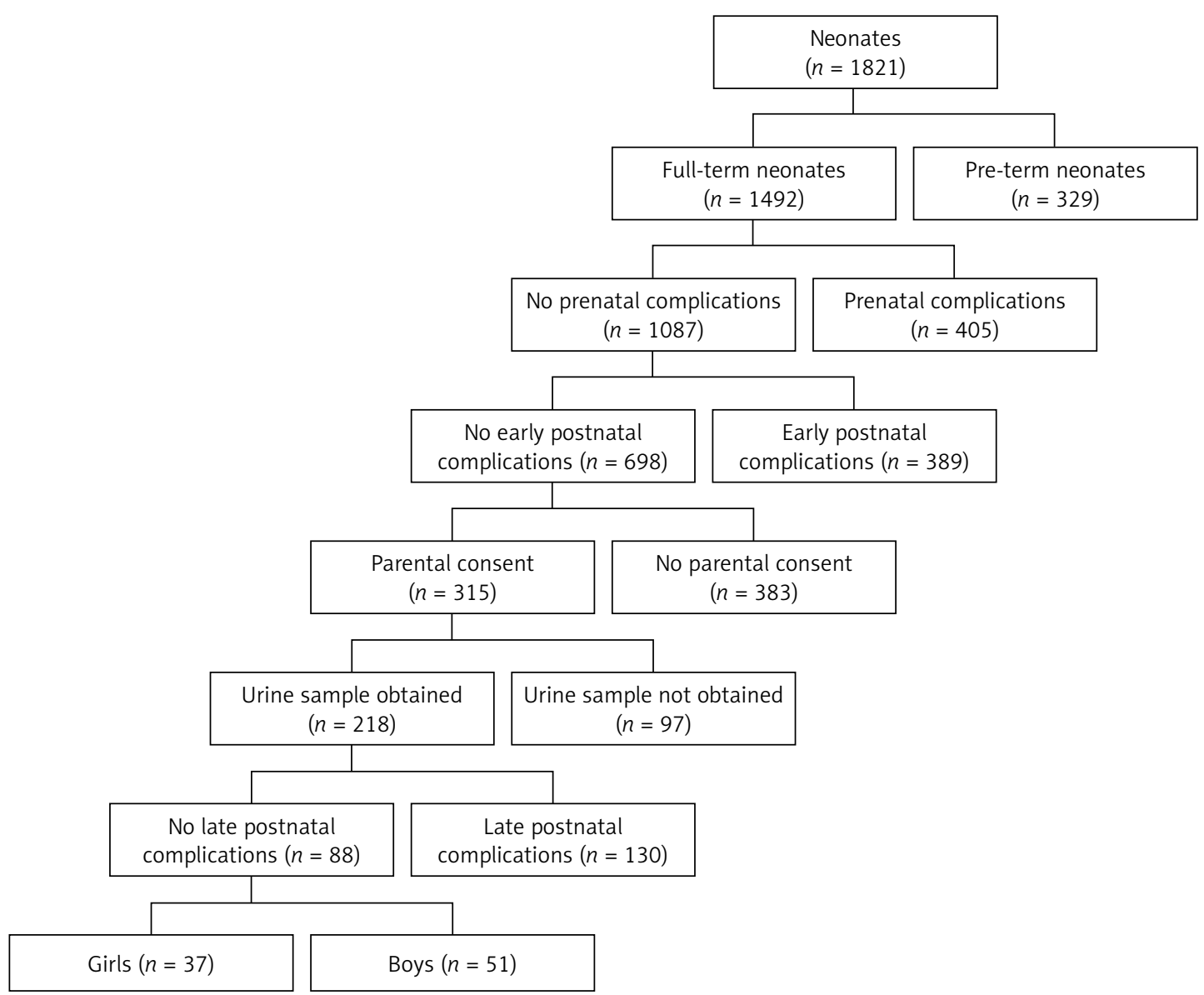

Figure 1. Study flow diagram. Prenatal complications: TORCH infection, coagulation disorders, hypertension, diabetes, antibiotic therapy in the third trimester of pregnancy, abnormal prenatal ultrasound examination. Early/ late postnatal complications: among others congenital anomaly, intrauterine growth retardation, Apgar's score $\leq 7$, intrauterine infections, elevated inflammatory markers, cardiovascular disorders, respiratory disorders, serological conflict, abnormal physical examination, digestive disorders, pathological jaundice

containing tetramethylbenzidine. The intensity of the color reaction was then measured at $450 \mathrm{~nm}$ with a microtiter plate reader and compared with a standard curve. Netrin-1 was expressed in nanograms per milliliter $(\mathrm{ng} / \mathrm{ml})$ and was normalized for urinary creatinine concentration determined by the enzymatic method to account for the potential confounding effect of urinary dilution, and expressed in nanograms per milligram of creatinine (netrin-1/crea ng/mg crea). Netrin-1 concentrations below the detection limit were recorded as $0.312 \mathrm{ng} / \mathrm{ml}$.

\section{Determination of serum cystatin C and creatinine}

Serum concentrations of cystatin C were determined using the human Cystatin C ELISA kit (BioVendor, Brno, Czech Republic) according to the manufacturer's instructions. Intra-assay and inter-assay variations of the test were 3.4 and $6.9 \%$, respectively. Cystatin $\mathrm{C}$ concentrations below the detection limit were recorded, as $0.25 \mathrm{ng} / \mathrm{ml}$. Se- rum concentration of creatinine was determined with the enzymatic method.

\section{Statistical analysis}

The results were analyzed with the Statistica 12.0 package (StatSoft, Tulsa, OK, USA). Discrete variables were expressed as counts (percentage), continuous variables as median and quartiles (Q1-Q3). The Shapiro-Wilk test was used to determine the normal distribution. The newborns were divided into subgroups depending on their gender (male and female) and the timing of urine sampling ( $1^{\text {st }}$ or $2^{\text {nd }}$ day of life). As the data were not normally distributed, the Mann-Whitney $U$-test was used for intergroup comparisons of continuous variables. The distributions of discrete variables were compared using the $\chi^{2}$ test or Fisher's exact test. The direction and power of association between the concentrations of netrin-1 and other variables were determined using Spearman's coefficients of rank correlation. The results of all the tests were considered significant at $p<0.05$. 
Table I. Median and quartiles (Q1-Q3) for urinary concentrations of netrin-1 and serum concentrations of cystatin $\mathrm{C}$ and creatinine in full-term healthy neonates, stratified according to their gender and timing of urine sampling

\begin{tabular}{|lccccc|}
\hline Group & Whole group & Females & Males & $1^{\text {st }}$ day of life & $2^{\text {nd }}$ day of life \\
\cline { 2 - 6 } & \multicolumn{5}{c}{ Median (Q1-Q3) } \\
\hline uNetrin-1 & 0.574 & 0.574 & 0.574 & 0.645 & 0.523 \\
[ng/ml] & $(0.438-0.896)$ & $(0.442-0.958)$ & $(0.426-0.786)$ & $(0.442-1.000)$ & $(0.426-0.738)$ \\
\hline uNetrin-1/crea & 0.771 & 0.744 & 0.776 & $0.622^{*}$ \\
[ng/mg crea $]$ & $(0.400-1.611)$ & $(0.419-1.454)$ & $(0.386-1.621)$ & $(0.495-3.107)$ & $(0.382-1.187)$ \\
\hline uCreatinine & 82.69 & 94.12 & 80.63 & 49.08 & $98.53^{*}(62.14-$ \\
[mg/ml] & $(47.43-123.97)$ & $(48.74-121.70)$ & $(44.35-124.80)$ & $(37.79-105.370)$ & $131.78)$ \\
\hline sCystatin C & 1729.46 & 1727.01 & 1762.60 & 1753.63 & $1719.20(1605.06-$ \\
[ng/ml] & $(1612.40-1954.29)$ & $(1613.32-1945.80)$ & $(1612.40-1960.53)$ & $(1613.32-1945.80)$ & $1954.29)$ \\
\hline sCreatinine & 0.780 & 0.740 & 0.805 & 0.780 & 0.760 \\
[mg/ml] & $(0.590-0.935)$ & $(0.580-0.820)$ & $(0.620-0.960)$ & $(0.620-0.960)$ & $(0.590-0.900)$ \\
\hline
\end{tabular}

${ }^{*} p$-comparison between the measurement at $1^{\text {st }}$ and $2^{\text {nd }}$ day of life $(p<0.05)$, crea - creatinine, sCystatin $C-\operatorname{serum} c y s t a t i n C$, sCreatinine - serum creatinine, uNetrin-1 - urinary netrin-1, uCreatinine - urinary creatinine.

\section{Results}

The study included 88 healthy full-term neonates (51 boys and 37 girls) with median birth weight of $3450 \mathrm{~g}(\mathrm{Q} 1-3185-\mathrm{Q} 3-3800 \mathrm{~g})$ and median gestational age of 40 weeks (Q1-38.5 Q3-40 weeks). In $40 \%$ of female and $47 \%$ of male neonates urine and serum samples were obtained on the $1^{\text {st }}$ day of life, and in the rest on the $2^{\text {nd }}$ day of life.

The analyzed subgroups (male and female newborns, children in whom urine samples were obtained on the $1^{\text {st }}$ and $2^{\text {nd }}$ day of life) did not differ significantly in terms of their gestational age and birth weights.

Urinary values of netrin-1, netrin-1/crea and serum concentrations of cystatin $C$ and creatinine are presented in Table I. We did not find significant differences in concentrations of any parameters between male and female newborns. The urinary concentration of netrin-1 was independent of the time of urine collection. After normalization for

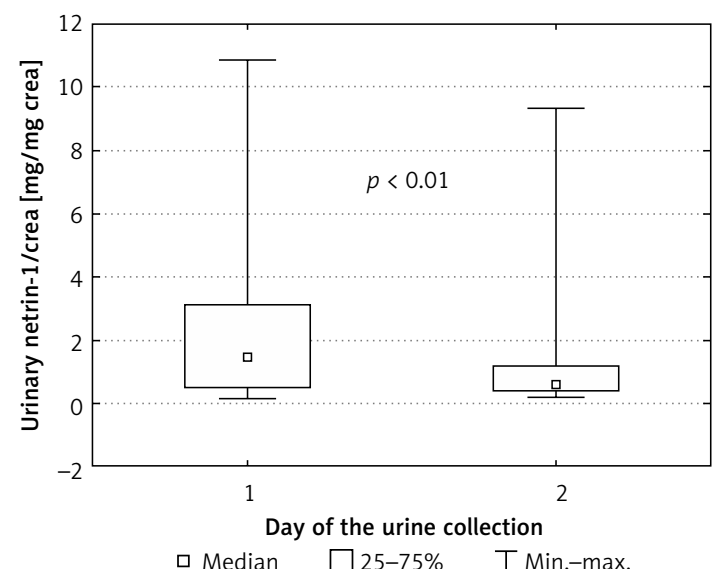

Figure 2. Netrin-1 concentration after normalization for urinary creatinine concentration depending on day of urine collection urinary creatinine, this difference gained statistical significance and the values of netrin-1/crea on the first day of life were higher than on the second day (Figure 2).

We also found statistically significant differences between urinary netrin-1/crea values depending on the week of pregnancy. The highest values of urinary netrin-1/crea were found in the $37^{\text {th }}$ and $41^{\text {st }}$ weeks of pregnancy (ANOVA, $p<0.05$ ) (Figure 3).

We also found a negative correlation between the netrin and netrin-1/crea and the birth weight $(r=-0.245, p=0.021$ and $r=-0.274, p<0.01$, respectively) (Figure 4).

The correlations between urinary netrin-1/crea and serum cystatin $C$ and serum creatinine were positive but not statistically significant.

\section{Discussion}

This prospective study was the first one to make a credible assessment of the normal range of urinary nerin- 1 in healthy full-term neonates, which

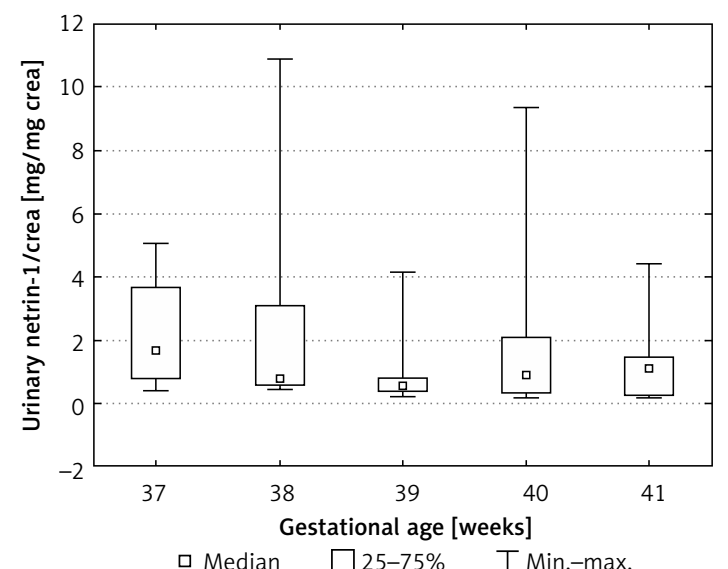

Figure 3. Netrin-1 concentration after normalization for urinary creatinine concentration depending on gestational age 
is considered a new early diagnostic biomarker of kidney injury. Based on previous studies, urinary netrin-1 most likely comes from proximal tubular epithelium of the kidney. The contribution of serum netrin-1 to urinary netrin-1 is unlikely due to its large size (72 kDa) [14]. Monitoring of renal function in acute kidney injury in the pediatric population is complicated by the lack of age-related reference values of new biomarkers. One of the main goals of this study was to present normal urinary netrin- 1 concentrations and to ascertain whether they correlated with well-known parameters of renal function (serum creatinine, serum cystatin C) and birth weight.

The reference ranges for urinary netrin-1 in healthy adults and children have not been published in the literature to date. The only data concerning the normal values of urine netrin-1 concentrations come from control groups from studies conducted in patients with renal dysfunctions $[12,13]$.

In this study, we found that urinary concentrations of netrin-1 did not differ in males and females, contrary for example to leptin concentration, which was found to be higher in female than male newborns, which suggests that sex hormones do not play a role in netrin secretion [15].

We found significantly higher values of urine netrin-1/crea in the first $24 \mathrm{~h}$, when compared to the second day of life, but this probably results from the increase in urine creatinine on the second day of life. Additionally we noted that the highest values of urinary netrin-1 were found in children born in the $37^{\text {th }}$ week of pregnancy. Statistically significant negative correlations between birth weight and urinary netrin-1, and netrin-1/ crea $(r=-0.245, p=0.021 ; r=-0.274, p=0.009$ respectively) were observed. In our opinion higher netrin-1 concentration in younger newborns and in newborns with lower birth weight may be an expression of the immaturity of the kidney tubules. In the study of Askenazi et al. baseline values of the tubular damage markers urinary neutrophil gelatinase associated lipocalin, (NGAL), kidney injury molecule-1 (KIM-1), $\beta 2$ microglobulin (B2mG) and cystatin-C (CysC) decrease with increasing gestational age [16]. Lavery et al. documented that UNGAL levels correlated negatively with both birth weight and gestational age [17]. In our future investigation, we plan to determine urinary netrin-1 concentration in the urine of premature infants and newborns treated with nephrotoxic drugs.

In this study the correlation between netrin-1/ crea and serum cystatin C and serum creatinine was positive but not statistically significant. We could not explain the lack of statistical significance, but it might be caused by the fact that serum creatinine concentrations and serum cystatin

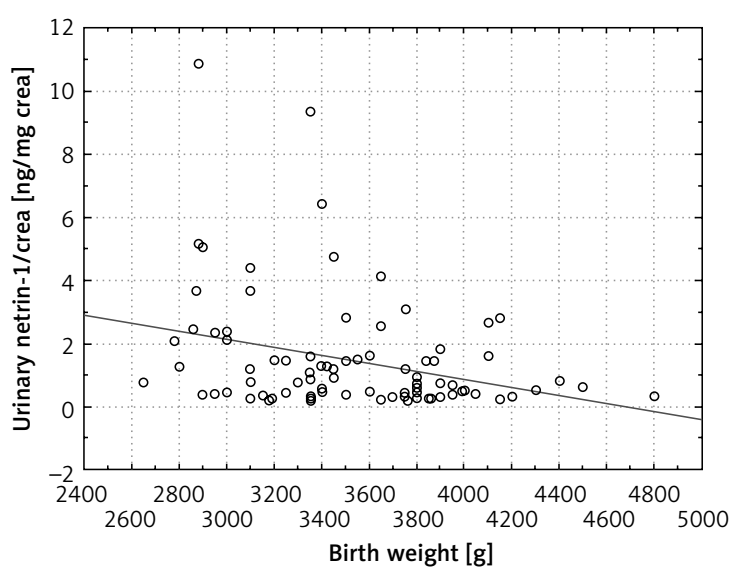

Figure 4. Correlation between urinary netrin-1/crea and birth weight of newborns

concentrations may reflect the mother's concentrations.

To the best of our knowledge there are very few studies reporting urinary netrin-1 concentrations in newborns $[12,13]$. In the study by Oncel et al. the concentrations of urinary netrin-1 on the first postnatal day were higher in newborns with perinatal asphyxia compared to controls. The concentration of urinary netrin-1 in a control group of 20 healthy, full term newborns at day 1 of life was (mean \pm SD) $592 \pm 181 \mathrm{pg} / \mathrm{ml}$ [12]

There are also only a few studies in older children $[9,18]$. A study by Övünç Hacıhamdioğlu et al. found that the value of urinary netrin $1 /$ crea in healthy controls was (mean \pm SD) 240.9 (123.5450.2) pg/mg crea [9]. Ramesh et al. in a study in patients after cardiopulmonary bypass gave the results of urinary netrin- 1 in healthy controls in the range $100-200 \mathrm{pg} / \mathrm{mg}$ crea [18].

In the adult population, there are only three studies reporting the urine concentrations of urinary netrin-1 $[8,14,19]$. Tu et al. presented the urinary concentrations of netrin- 1 in the control group of 50 healthy subjects aged $56 \pm 11$ years at the level of (mean \pm SD) $2.50 \pm 1.46 \mathrm{ng} / \mathrm{l}$ but the values were not normalized for urinary creatinine [19]. In the study by Reeves et al. netrin-1 was undetectable by Western blot analysis in the urine of six normal volunteers [14]. Jayakumar et al. reported that the concentration of urinary netrin- 1 in the control group of 42 healthy volunteers aged 53.3 \pm 13.1 years, presented as the median (min.-max.) value, was 430 (229-1087) pg/ml. Our study has some limitations. It is a single-center study with a relatively small sample size. A larger study with more subjects needs to be conducted to assess the variation in urinary netrin-1 levels in full term and pre-term neonates.

This is the first study showing urinary netrin-1 concentration in healthy full-term newborns. Future investigation is needed to confirm its potential role as a marker of kidney function in this age group. 


\section{Acknowledgments}

This study was funded by a grant of the Medical University of Białystok.

\section{Conflict of interest}

The authors declare no conflict of interest.

\section{References}

1. Dun XP, Parkinson DB. Role of netrin-1 signaling in nerve regeneration. Int J Mol Sci 2017; 18: 491.

2. Mehlen P, Furne C. Netrin-1: when a neuronal guidance cue turns out to be a regulator of tumorigenesis. Cell Mol Life Sci 2005; 62: 2599-616.

3. Boneschansker L, Nakayama H, Eisenga $\mathrm{M}$, et al. Netrin-1 augments chemokinesis in CD4+ T cells in vitro and elicits a proinflammatory response in vivo. I Immunol 2016; 197: 1389-98.

4. Prieto CP, Ortiz MC, Villanueva A, et al. Netrin-1 acts as a non-canonical angiogenic factor produced by human Wharton's jelly mesenchymal stem cells (WJ-MSC). Stem Cell Res Ther 2017; 8: 43.

5. Wang W, Reeves WB, Ramesh G. Netrin-1 and kidney injury. I. Netrin-1 protects against ischemia-reperfusion injury of the kidney. Am J Physiol Renal Physiol 2008; 294: F739-47.

6. White JJ, Mohamed R, Jayakumar C, Ramesh G. Tubular injury marker netrin- 1 is elevated early in experimental diabetes. J Nephrol 2013; 26: 1055-64.

7. Mussap M, Noto A, Fravega M, Fanos V. Urine neutrophil gelatinase-associated lipocalin (UNGAL) and netrin-1: are they effectively improving the clinical management of sepsis-induced acute kidney injury (AKI)? J Matern Fetal Neonatal Med 2011; 24 Suppl 2: 15-7.

8. Jayakumar C, Nauta FL, Bakker SJ, et al. Netrin-1, a urinary proximal tubular injury marker, is elevated early in the time course of human diabetes. J Nephrol 2014; 27: 151-7.

9. Övünç Hacıhamdioğlu D, Hacıhamdioğlu B, Altun D, Müftüoğlu T, Karademir F, Süleymanoğlu S. Urinary netrin-1: a new biomarker for the early diagnosis of renal damage in obese children. J Clin Res Pediatr Endocrinol 2016; 8: 282-7.

10. Libório AB, Branco KM, Torres de Melo Bezerra C. Acute kidney injury in neonates: from urine output to new biomarkers. Biomed Res Int 2014; 2014: 601568.

11. Stritzke A, Thomas S, Amin H, Fusch C, Lodha A. Renal consequences of preterm birth. Mol Cell Pediatr 2017. 4: 2.

12. Oncel MY, Canpolat FE, Arayici S, Alyamac Dizdar E, Uras N, Oguz SS. Urinary markers of acute kidney injury in newborns with perinatal asphyxia. Ren Fail 2016; 38: 882-8.

13. Cao XY, Zhang HR, Zhang W, Chen B. Diagnostic values of urinary netrin-1 and kidney injury molecule-1 for acute kidney injury induced by neonatal asphyxia. Zhongguo Dang Dai Er Ke Za Zhi 2016; 18: 24-8.

14. Reeves WB, Kwon O, Ramesh G. Netrin-1 and kidney injury. II. Netrin-1 is an early biomarker of acute kidney injury. Am J Physiol Renal Physiol 2008; 294: F731-8.

15. Biesiada LA, Głowacka E, Krekora M, Sobantka S, Krokocka A, Krasomski G. The impact of excessive maternal weight on the nutritional status of the fetus - the role of leptin. Arch Med Sci 2016; 12: 394-401.
16. Askenazi DJ, Koralkar R, Levitan EB, et al. Baseline values of candidate urine acute kidney injury biomarkers vary by gestational age in premature infants. Pediatr Res 2011; 70: 302-6.

17. Lavery AP, Meinzen-Derr JK, Anderson E, et al. Urinary NGAL in premature infants. Pediatr Res 2008; 64: 423-8.

18. Ramesh G, Krawczeski CD, Woo JG, Wang Y, Devarajan P. Urinary netrin-1 is an early predictive biomarker of acute kidney injury after cardiac surgery. Clin J Am Soc Nephrol 2010; 5: 395-401.

19. Tu Y, Wang H, Sun R, et al. Urinary netrin-1 and KIM-1 as early biomarkers for septic acute kidney injury. Ren Fail 2014; 36: 1559-63. 